\title{
Prevendo as Complicações do Implante Percutâneo Valvar Aórtico: Hora de Ir Além da Estratégia do "Esperar para Ver"
}

\author{
Ver artigos relacionados \\ nas páginas 128 e 135
}

\author{
Francesco Saia ${ }^{1}$
}

$\mathrm{N}$ esta edição da Revista Brasileira de Cardiologia Invasiva, Brito Jr. et al. ${ }^{1}$ e Lemos et al. ${ }^{2}$ relatam suas respectivas experiências sobre a incidência de distúrbios da condução elétrica do coração após implante percutâneo valvar aórtico com a prótese CoreValve (Medtronic, Minneapolis, Estados Unidos). Os autores devem ser elogiados pela tentativa de esclarecer um problema já conhecido do implante percutâneo valvar aórtico, porém de mecanismo ainda obscuro. Um bloqueio atrioventricular súbito após o procedimento pode ser uma complicação fatal e tem sido aventada como possível causa de morte súbita, tanto na fase hospitalar como no acompanhamento. É necessário melhorar nossa capacidade de prever os distúrbios da condução elétrica, tanto no pré-procedimento, pois podemos mudar de estratégia com base nessa informação, como no período pós-procedimento, de forma a identificarmos os pacientes com necessidade de monitoramento eletrocardiográfico prolongado.

Num primeiro momento, a mensagem principal que tiramos desses dois relatos não parece muito reconfortante: com o conhecimento de que dispomos atualmente, ainda não conseguimos prever a ocorrência de distúrbios da condução elétrica e a necessidade de implante de marca-passo definitivo após implante percutâneo valvar aórtico. De fato, na pequena série de pacientes relatados por Lemos et al. ${ }^{2}$, nenhum dos pacientes necessitou de implante de marca-passo definitivo, apesar de a maioria dos casos ter apresentado pelo menos uma característica de alto risco para bloqueio atrioventricular pós-procedimento. Já o relato de Brito Jr. et al. ${ }^{1}$ é mais consistente com a literatura, demonstrando $30 \%$ de novos implantes de marcapasso definitivo e cerca de $50 \%$ de bloqueios de ramo esquerdo pós-procedimento. Entretanto, mesmo nessa série há elementos de incerteza. Ao contrário de publicações anteriores, ${ }^{3-5}$ os autores não encontraram rela- ção entre profundidade do implante de bioprótese na via de saída do ventrículo esquerdo e distúrbios da condução elétrica, nem entre a razão prótese/anel e a ocorrência de distúrbios da condução elétrica. Essa observação não é meramente acadêmica, pois sua consequência direta é que não é possível esperar resultados mais previsíveis (e melhores) com uma seleção mais acurada do paciente e do dispositivo ou com um melhor implante (ou seja, menos profundo) da prótese.

O interessante é que uma leitura mais profunda desses artigos, em conjunto com outras experiências, nos revela um lado otimista, representado pelo ganho progressivo de conhecimento, e para o qual esses artigos certamente contribuem. Primeiro, a necessidade de implante de marca-passo definitivo parece ser claramente maior com o dispositivo CoreValve da Medtronic que com o Edwards-Sapien (Edwards Lifesciences Inc., Irvine, Estados Unidos).5,6 Sugere-se que o perfil mais longo da prótese e a propriedade intrínseca do nitinol, cuja expansão continua horas após o implante, poderia ser a causa de maior trauma às estruturas da via de condução elétrica do coração. Após o implante transcateter da prótese balão-expansível Edwards-Sapien, a incidência de bloqueio de condução atrioventricular necessitando de implante de marca-passo definitivo varia de 0 a $6 \%$, e a incidência de novo bloqueio de ramo esquerdo é de aproximadamente $3 \% .^{7-9}$

Em segundo lugar, o estudo de Brito Jr. et al.' reforça a observação de que o bloqueio de ramo direito preexistente pode ser um forte preditor de implante de marcapasso definitivo após o procedimento, ${ }^{3,6}$ tem bom fundamento e pode refletir um fenômeno já descrito para os pacientes submetidos a cirurgia valvular convencional. ${ }^{10} \mathrm{~A}$ aparente irrelevância desse parâmetro em outros estudos pode ser casual; por exemplo, no relato do grupo de Munique, o bloqueio de ramo direito foi

\footnotetext{
1 Instituto de Cardiologia - Universidade de Bolonha - Policlinico S. Orsola-Malpighi - Bolonha, Itália

Correspondência: Francesco Saia. Via Massarenti, 9 - 40138 - Bologna, Italia

E-mail: francescosaia@hotmail.com

Recebido em: 20/6/2010 • Aceito em: 21/6/2010
} 
raro $(4 \%)$, e isso pode ser o motivo de não ter sido identificado como preditor de eventos na análise multivariada. ${ }^{5}$ É notável que metade dos pacientes com bloqueio de ramo direito tendeu a desenvolver bloqueio atrioventricular completo $(P=0,078)$. Da mesma forma, no estudo do grupo de Leicester apenas um paciente tinha bloqueio de ramo direito basal e necessitou de implante de marca-passo definitivo no primeiro dia após o procedimento. ${ }^{11}$ No estudo de Lemos et al. ${ }^{2}$ nenhum paciente com bloqueio de ramo direito desenvolveu bloqueio atrioventricular completo, mas deve-se reconhecer que houve apenas 3 pacientes com essa condição e que não se pode tirar uma conclusão definitiva com base nesses resultados.

Um terceiro e talvez mais importante ponto que podemos concluir por meio de uma visão integrada dos dois estudos e da literatura existente é que a necessidade de um marca-passo é provavelmente multifatorial e envolve vários fatores anatômicos, eletrocardiográficos e relacionados ao procedimento (Tabela 1). Essa parece ser a melhor forma de reconciliar os achados de Brito Jr. et al. ${ }^{1}$ e Lemos et al. ${ }^{2}$ de que não há relação entre profundidade do implante e razão prótese/anel com a necessidade de implante de marca-passo definitivo. É possível que esses elementos isoladamente não sejam suficientes e possam interagir com outros na gênese do distúrbio da condução elétrica, como desvio do eixo para a esquerda, hipertrofia septal grave, espessura da cúspide não-coronária e calcificação da zona de ancoragem do dispositivo. ${ }^{13}$ Além disso, não podemos descartar um possível papel de outros fatores não avaliados ou que ainda não foram identificados. Na verdade, observamos que a distância média entre a borda inferior da cúspide não-coronária até a extremidade ventricular da prótese foi bem profunda na série de Lemos et al. ${ }^{2}(14,1 \pm 2,9 \mathrm{~mm})$, em que não houve necessidade de implante de marca-passo definitivo, um pouco menos pronunciada na coorte de

TABELA 1

Incidência de bloqueio de ramo esquerdo novo e necessidade de implante de marca-passo permanente após implante percutâneo valvar aórtico com a prótese CoreValve

\begin{tabular}{|c|c|c|c|c|}
\hline Estudo & $\mathbf{n}$ & $\begin{array}{c}\text { BRE } \\
\text { novo } \\
(\%)\end{array}$ & $\begin{array}{l}\text { IMP } \\
(\%)\end{array}$ & Preditores de IMP \\
\hline Piazza et al. ${ }^{3}$ & 40 & 40 & 18 & - Bloqueio de ramo direito preexistente (observação) \\
\hline Calvi et al. ${ }^{12}$ & 30 & 46 & 20 & $-N D$ \\
\hline Jilaihawi et al. ${ }^{11}$ & 30 & 39 & 33 & $\begin{array}{l}\text { - Desvio do QRS para a esquerda no ECG basal } \\
\text { - BRE com desvio do eixo para a esquerda } \\
\text { - Dimensão do septo interventricular na diástole } \\
>17 \text { mm no ecocardiograma transtorácico } \\
\text { - Espessura da cúspide não-coronária }>8 \mathrm{~mm}\end{array}$ \\
\hline Erkapic et al. ${ }^{6}$ & $50^{*}$ & 30 & 34 & - Bloqueio completo do ramo direito \\
\hline CoreValve & 36 & ND & 44 & - CoreValve \\
\hline Bleiziffer et al. ${ }^{5}$ & $159 *$ & ND & 22 & - Mismatch válvula/anel \\
\hline CoreValve & 123 & ND & 27 & $\begin{array}{l}\text { - CoreValve } \\
\text { - Episódio de bloqueio AV imediato durante o implante }\end{array}$ \\
\hline \multirow[t]{2}{*}{ Baan et al. ${ }^{4}$} & 34 & 64 & 21 & $\begin{array}{l}\text { - Menor diâmetro da via de saída do ventrículo esquerdo } \\
\text { - Eixo do QRS com maior desvio para a esquerda } \\
\text { - Maior calcificação do anel mitral } \\
\text { - Menor área de orifício efetiva indexada pós-implante }\end{array}$ \\
\hline & & & & $\begin{array}{l}\text { Preditor de novo BRE } \\
\text { - Implante mais profundo da prótese }\end{array}$ \\
\hline Latsios et al. ${ }^{13}$ & 67 & ND & $31^{+}$ & $\begin{array}{l}\text { - Sexo feminino } \\
\text { - Fração de ejeção do VE reduzida } \\
\text { - Calcificação da zona de ancoragem da prótese } \\
\text { (escore de Agaston) }\end{array}$ \\
\hline
\end{tabular}

* Próteses CoreValve e Edwards-Sapien.

${ }^{+}$Porcentual de pacientes com indicação absoluta; 47\% efetivamente implantados.

$\mathrm{AV}=$ atrioventricular; $\mathrm{BRE}=$ bloqueio de ramo esquerdo; $\mathrm{IMP}=$ implante de marcapasso permanente; $\mathrm{n}=$ número de pacientes; $\mathrm{ND}=$ não disponível; $\mathrm{VE}$ = ventrículo esquerdo. 
Brito Jr. et al. ${ }^{1}$, em que não houve diferença significativa entre os pacientes que necessitaram ou não de implante de marca-passo definitivo $(13,4 \pm 1,1 \mathrm{~mm}$ vs. $12,5 \pm 3,5 \mathrm{~mm}$, respectivamente), e diferente do que foi descrito pelo grupo de Amsterdam (10,2 + 2,3 mm no grupo com bloqueio de ramo esquerdo novo vs. $7,7 \pm 3,1 \mathrm{~mm}$ no grupo sem bloqueio de ramo esquerdo; $\mathrm{P}=0,02) .{ }^{4}$ Portanto, temos que concordar com Lemos et al. ${ }^{2}$ de que "a necessidade de marcapasso após implante percutâneo valvar aórtico não é prontamente previsível com as características de alto risco descritas atualmente". Assim não devemos aceitar mais o provérbio "só a experiência comprova". Precisamos mais.

Com mais de 10 mil pacientes tratados no mundo todo com um dos dois dispositivos disponíveis, o implante percutâneo valvar aórtico já representa uma opção terapêutica viável e válida para pacientes com estenose aórtica sintomática grave, com alto risco para cirurgia de troca valvar aórtica. Houve melhora da classificação funcional (New York Heart Association NYHA) e da qualidade de vida dos pacientes que sobreviveram, demonstrando um benefício definitivo do tratamento no bem-estar físico e mental e no desempenho de atividades da vida cotidiana. ${ }^{14}$ Juntamente com o sabor desse sucesso, entretanto, vem o conhecimento das complicações relacionadas ao implante percutâneo valvar aórtico. ${ }^{15}$ Complicações relacionadas ao local de acesso, distúrbios de condução, complicações cerebrais isquêmicas, oclusão aguda da coronária e lesão da válvula mitral são eventos preocupantes que devem ser extensivamente investigados e possivelmente evitados. O extravasamento paravalvar residual ${ }^{15}$ e novos focos de isquemia cerebral clinicamente silenciosos ${ }^{16}$ são frequentes e sua significância prognóstica é desconhecida. Um novo e persistente bloqueio de ramo esquerdo adquirido após a troca cirúrgica da válvula aórtica foi identificado como forte preditor independente de evento adverso durante o acompanhamento. ${ }^{17}$ Será que isso também aconteceria após o implante percutâneo valvar aórtico? Um progresso substancial já foi alcançado e a prótese e os sistemas de liberação estão passando por rápida evolução. Este é um período de transição do uso por compaixão em pacientes extremamente graves para o uso em pacientes de menor risco. Enquanto aguardamos os resultados de estudos randomizados e registros pós-marketing maiores, devemos evitar ampliar as indicações do implante percutâneo valvar aórtico enquanto não conhecermos profundamente suas consequências.

\section{CONFLITO DE INTERESSES}

O autor declarou inexistência de conflito de interesses relacionado a este manuscrito.

\section{REFERÊNCIAS}

1. Brito Jr. FS, Perin M, Almeida BO, Abizaid A, Pereira MAM, Caixeta $A$, et al. Incidência de distúrbios da condução atrioventricular e intraventricular após implante percutâneo da bioprótese valvar aórtica CoreValve. Rev Bras Cardiol Invasiva. 2010;18(2):128-34.

2. Lemos PA, Mariani Jr J, Esteves Filho A, Kajita LJ, Cardoso LF, Dracoulakis MDA, et al. Implante transcateter de valva aórtica sem a necessidade de marca-passo definitivo em uma série de casos consecutivos: é possível predizer o risco de bloqueio atrioventricular? Rev Bras Cardiol Invasiva. 2010; 18(2):135-9.

3. Piazza N, Onuma Y, Jesserun E, Kint PP, Maugenest AM, Anderson RH, et al. Early and persistent intraventricular conduction abnormalities and requirements for pacemaking after percutaneous replacement of the aortic valve. JACC Cardiovasc Interv. 2008;1(3):310-6.

4. Baan J Jr, Yong ZY, Koch KT, Henriques JP, Bouma BJ, Vis $\mathrm{MM}$, et al. Factors associated with cardiac conduction disorders and permanent pacemaker implantation after percutaneous aortic valve implantation with the CoreValve prosthesis. Am Heart J. 2010;159(3):497-503.

5. Bleiziffer S, Ruge H, Horer J, Hutter A, Geisbusch S, Brockmann G, et al. Predictors for new-onset complete heart block after transcatheter aortic valve implantation. JACC Cardiovasc Interv. 2010;3(5):524-30.

6. Erkapic D, Kim WK, Weber M, Mollmann H, Berkowitsch A, Zaltsberg S, et al. Electrocardiographic and further predictors for permanent pacemaker requirement after transcatheter aortic valve implantation. Europace. 2010 Mar 30. [Epub ahead of print].

7. Webb JG, Pasupati S, Humphries K, Thompson C, Altwegg L, Moss $R$, et al. Percutaneous transarterial aortic valve replacement in selected high-risk patients with aortic stenosis. Circulation. 2007;116(7):755-63.

8. Sinhal A, Altwegg L, Pasupati S, Humphries $\mathrm{KH}$, Allard $\mathrm{M}$, Martin P, et al. Atrioventricular block after transcatheter balloon expandable aortic valve implantation. JACC Cardiovasc Interv. 2008;1(3):305-9.

9. Walther T, Falk V, Kempfert J, Borger MA, Fassl J, Chu MW, et al. Transapical minimally invasive aortic valve implantation; the initial 50 patients. Eur J Cardiothorac Surg. 2008;33(6): 983-8.

10. Koplan BA, Stevenson WG, Epstein LM, Aranki SF, Maisel $\mathrm{WH}$. Development and validation of a simple risk score to predict the need for permanent pacing after cardiac valve surgery. J Am Coll Cardiol. 2003;41(5):795-801.

11. Jilaihawi $H$, Chin D, Vasa-Nicotera $M$, Jeilan $M$, Spyt $T, N g$ $G A$, et al. Predictors for permanent pacemaker requirement after transcatheter aortic valve implantation with the CoreValve bioprosthesis. Am Heart J. 2009;157(5):860-6.

12. Calvi V, Puzzangara E, Pruiti GP, Conti S, Di Grazia A, Ussia $\mathrm{GP}$, et al. Early conduction disorders following percutaneous aortic valve replacement. Pacing Clin Electrophysiol. 2009; 32 Suppl 1:S126-30.

13. Latsios G, Gerckens U, Buellesfeld L, Mueller R, John D, Yuecel S, et al. "Device landing zone" calcification, assessed by MSCT, as a predictive factor for pacemaker implantation after TAVI. Catheter Cardiovasc Interv. 2010 Mar 26. [Epub ahead of print].

14. Ussia GP, Mule M, Barbanti M, Cammalleri V, Scarabelli M, Imme $S$, et al. Quality of life assessment after percutaneous aortic valve implantation. Eur Heart J. 2009;30(14):1790-6.

15. Masson JB, Kovac J, Schuler G, Ye J, Cheung A, Kapadia S, et al. Transcatheter aortic valve implantation: review of the nature, management, and avoidance of procedural complications. JACC Cardiovasc Interv. 2009;2(9):811-20.

16. Kahlert P, Knipp SC, Schlamann M, Thielmann M, Al-Rashid 
Saia F. Prevendo as Complicações do Implante Percutâneo Valvar Aórtico: Hora de Ir Além da Estratégia do "Esperar para Ver". Rev Bras Cardiol Invasiva. 2010;18(2):119-22.

F, Weber M, et al. Silent and apparent cerebral ischemia after percutaneous transfemoral aortic valve implantation: a diffusionweighted magnetic resonance imaging study. Circulation. 2010;121(7):870-8.
17. El-Khally Z, Thibault B, Staniloae C, Theroux P, Dubuc M, Roy $\mathrm{D}$, et al. Prognostic significance of newly acquired bundle branch block after aortic valve replacement. Am J Cardiol. 2004;94(8):1008-11. 